\title{
Quantum Monte Carlo Calculation of the Binding Energy of Bilayer Graphene
}

\author{
E. Mostaani and N. D. Drummond \\ Department of Physics, Lancaster University, Lancaster LA1 4YB, United Kingdom \\ V. I. Fal'ko \\ Department of Physics, Lancaster University, Lancaster LA1 4YB, United Kingdom and \\ National Graphene Institute, University of Manchester, \\ Booth Street East, Manchester M13 9PL, United Kingdom
}

(Dated: September 22, 2015)

\begin{abstract}
We report diffusion quantum Monte Carlo calculations of the interlayer binding energy of bilayer graphene. We find the binding energies of the AA- and AB-stacked structures at the equilibrium separation to be $11.5(9)$ and $17.7(9) \mathrm{meV} /$ atom, respectively. The out-of-plane zone-center optical phonon frequency predicted by our binding-energy curve is consistent with available experimental results. As well as assisting the modeling of interactions between graphene layers, our results will facilitate the development of van der Waals exchange-correlation functionals for density functional theory calculations.
\end{abstract}

PACS numbers: 61.48.Gh, 71.15.Nc, 02.70.Ss

van der Waals (vdW) interactions play a crucial role in a wide range of physical and biological phenomena, from the binding of rare-gas solids to the folding of proteins. Significant efforts are therefore being made to develop computational methods that predict vdW contributions to energies of adhesion, particularly for materials such as multilayer graphene. This task has proved to be challenging, however, because vdW interactions are caused by nonlocal electron correlation effects. Standard first-principles approaches such as density functional theory (DFT) with local exchange-correlation functionals do not describe vdW interactions accurately. One technique for including vdW interactions in a first-principles framework is to add energies obtained using pairwise interatomic potentials to DFT total energies; this is the so-called DFT-D scheme [1 4]. The development of vdW density functionals (vdW-DFs) that can describe vdW interactions in a seamless fashion is another promising approach [5]. DFT-based random-phase approximation (RPA) calculations of the correlation energy [9, 10 provide a more sophisticated method for treating vdW interactions; however, RPA atomization energies are typically overestimated by up to $15 \%$ for solids [11, 12], and hence the accuracy of this approach is unclear. Symmetry-adapted perturbation theory based on DFT allows one to calculate the vdW interactions between molecules and hence, by extrapolation, between nanostructures [13. Finally, empirical interatomic potentials with $r^{-6}$ tails may be used to calculate binding energies 14, 15, although such potentials give a qualitatively incorrect description of the interaction of metallic or $\pi$ bonded two-dimensional (2D) materials at large separation [16].

A key test system for methods purporting to describe $\mathrm{vdW}$ interactions between low-dimensional materials is bilayer graphene (BLG). Several theoretical studies have
TABLE I: BE of BLG (both AA- and AB-stacked) obtained in recent theoretical studies. The layer separations $d$ quoted in the table are the ones used in the calculations, not necessarily the optimized bond length for the given method. "SAPT(DFT)" and "DFT-LCAO-OO" denote symmetryadapted perturbation theory based on DFT and linear combination of atomic orbitals-orbital occupancy based on DFT, respectively. "MBD" denotes many-body dispersion calculations.

\begin{tabular}{lclc}
\hline \hline Stacking & Method & $d(\AA)$ & $\mathrm{BE}(\mathrm{meV} /$ atom $)$ \\
\hline AA & vdW-DF [17] & 3.35 & 10.4 \\
AA & DFT-D [17] & 3.25 & 31.1 \\
AA & DMC (pres. wk.) & 3.495 & $11.5(9)$ \\
AB & DFT-LCAO-OO [18 & $3.1-3.2$ & $70(5)$ \\
AB & SAPT(DFT) [19] & 3.43 & 42.5 \\
AB & vdW-DF [7] & 3.6 & 45.5 \\
AB & vdW-DF [17] & 3.35 & 29.3 \\
AB & DFT-D [17] & 3.25 & 50.6 \\
AB & DFT-D [20] & 3.32 & 22 \\
AB & MBD [21] & 3.37 & 23 \\
AB & DMC (pres. wk.) & 3.384 & $17.7(9)$ \\
\hline \hline
\end{tabular}

used methods based on DFT to calculate the binding energy (BE) of BLG. Some of the results are summarized in Table I] but there is very little consensus. In this work we provide diffusion quantum Monte Carlo (DMC) data for the BE of BLG and the atomization energy of monolayer graphene (MLG), which we have extrapolated to the thermodynamic limit. We find the DMC BE of BLG to be somewhat less than the BEs predicted by DFT-D, although the latter vary significantly from scheme to scheme. The DMC method is the most accurate first-principles technique available for studying condensed matter. Our data can therefore be used as a benchmark for the development of vdW functionals. 
We have used the variational quantum Monte Carlo and DMC methods as implemented in the CAsino code 22] to study MLG and BLG. In the former method, Monte Carlo integration is used to evaluate expectation values with respect to trial many-body wave-function forms that may be of arbitrary complexity. In the DMC method [23, 24, a stochastic process governed by the Schrödinger equation in imaginary time is simulated to project out the ground-state component of the trial wave function. Fermionic antisymmetry is maintained by the fixed-node approximation, in which the nodal surface is constrained to equal that of the trial wave function 25. DMC methods have recently been used to study the BE of hexagonal boron nitride bilayers [26].

Our many-body trial wave-function form consisted of Slater determinants for spin-up and spin-down electrons multiplied by a symmetric, positive Jastrow correlation factor $\exp (J)$ 24. The Slater determinants contained Kohn-Sham orbitals that were generated using the CASTEP plane-wave DFT code [27] within the local density approximation (LDA). We performed test DMC calculations for $3 \times 3$ supercells of MLG and AB-stacked BLG using Perdew-Burke-Ernzerhof (PBE) [28] orbitals. The effect of changing the orbitals on the DMC total energies (and hence the BE) was statistically insignificant.

To improve the scaling of our DMC calculations and to allow the use of 2D-periodic boundary conditions, the orbitals were re-represented in a B-spline (blip) basis [29]. The Jastrow exponent $J$ consisted of polynomial and plane-wave expansions in the electron-ion and electron-electron distances [30. The free parameters in the Jastrow factor were optimized by unreweighted variance minimization [31, 32. The DMC energy was extrapolated linearly to zero time step and we verified that finite-population errors in our results are negligible 33 . The fixed-node error is of uncertain magnitude, but it is always positive, and should largely cancel when the $\mathrm{BE}$ is calculated. We used Dirac-Fock pseudopotentials to represent the $\mathrm{C}$ atoms 34, 35 and fixed the in-plane lattice parameter at the experimental value of $a=2.460$ A.

The principal source of uncertainty in our BE results is the need to use finite simulation cells subject to periodic boundary conditions in DMC calculations for condensed matter. Finite-size errors in DMC total energies consist of (i) pseudorandom, oscillatory single-particle finitesize errors due to momentum quantization and (ii) systematic finite-size errors due to the inability to describe long-range two-body correlations and the difference between $1 / r$ and the 2D Ewald interaction [36, 37] in a finite periodic cell. By dividing the electron-electron interaction energy into a Hartree term (the electrostatic energy of the charge density) and an exchange-correlation energy (the interaction energy of each electron with its accompanying exchange-correlation hole) and considering the long-range nonoscillatory behavior of the hole pre- dicted by the RPA, it can be shown that the systematic finite-size error in the interaction energy per electron of a 2D-periodic system is negative and scales asymptotically with system size $N$ as $O\left(N^{-5 / 4}\right)$ 38. The leading-order long-range finite-size error in the kinetic energy per electron behaves in a similar fashion. The finite-size error in the atomization energy is therefore positive and scales as $O\left(N^{-5 / 4}\right)$, and the finite-size error in the BE per atom must also exhibit the $O\left(N^{-5 / 4}\right)$ scaling. We also investigated finite-size errors in the asymptotic BE using the Lifshitz theory of vdW interactions [39, 40, with a Dirac model of electron dispersion in graphene. To study finite system sizes, we introduced a cutoff wavelength that depended on the cell size and layer separation. However, near the equilibrium separation, short-range interactions are important and the contribution to the finite-size error from the Lifshitz theory is negligible. In order to eliminate finite-size effects and obtain the atomization and binding energies in the thermodynamic limit, we studied simulation cells consisting of arrays of $3 \times 3,4 \times 4$, and $6 \times 6$ primitive cells for MLG and BLG at the equilibrium layer separation and $3 \times 3$ and $5 \times 5$ cells for BLG at nonequilibrium layer separations. We used canonicalensemble twist averaging [41] (i.e., averaging over offsets to the grid of $\mathbf{k}$ vectors) to reduce the oscillatory singleparticle finite-size errors in the ground-state energies of MLG and BLG. To obtain the twist-averaged energy of MLG in a simulation cell containing $N_{P}$ primitive cells, we performed DMC calculations at twelve random offsets $\mathbf{k}_{s}$ to the grid of $\mathbf{k}$ vectors, then fitted

$$
E\left(N_{P}, \mathbf{k}_{s}\right)=\bar{E}\left(N_{P}\right)+b\left[E_{\mathrm{LDA}}\left(N_{P}, \mathbf{k}_{s}\right)-E_{\mathrm{LDA}}(\infty)\right]
$$

to the DMC energies per atom $E\left(N_{P}, \mathbf{k}_{s}\right)$. The model function has two fitting parameters: $\bar{E}\left(N_{P}\right)$, which is the twist-averaged DMC energy per atom, and $b$. $E_{\mathrm{LDA}}\left(N_{P}, \mathbf{k}_{s}\right)$ is the DFT-LDA energy per atom of MLG obtained using the offset $\mathbf{k}$-point grid corresponding to the supercell used in the DMC calculations, and $E_{\mathrm{LDA}}(\infty)$ is the DFT-LDA energy per atom obtained using a fine $(50 \times 50) \mathbf{k}$-point mesh. Finally, we extrapolated our total-energy data to infinite system size by fitting

$$
\bar{E}\left(N_{P}\right)=E(\infty)+c N_{P}^{-5 / 4}
$$

to the twist-averaged energies per atom, where the extrapolated energy per atom $E(\infty)$ and $c$ are fitting parameters. The atomization energy of MLG is the difference between the energy of an isolated, spin-polarized $\mathrm{C}$ atom and the energy per atom of MLG.

Our DMC atomization energies of MLG as a function of system size are plotted in Fig. 1. We find the staticnucleus DMC atomization energy to be $7.395(3) \mathrm{eV} /$ atom with a Slater-Jastrow trial wave function. This is lower than the DMC result of 7.464(10) eV/atom reported in Ref. 42. Most of this disagreement arises from the use of different pseudopotentials in the two works [33. The 


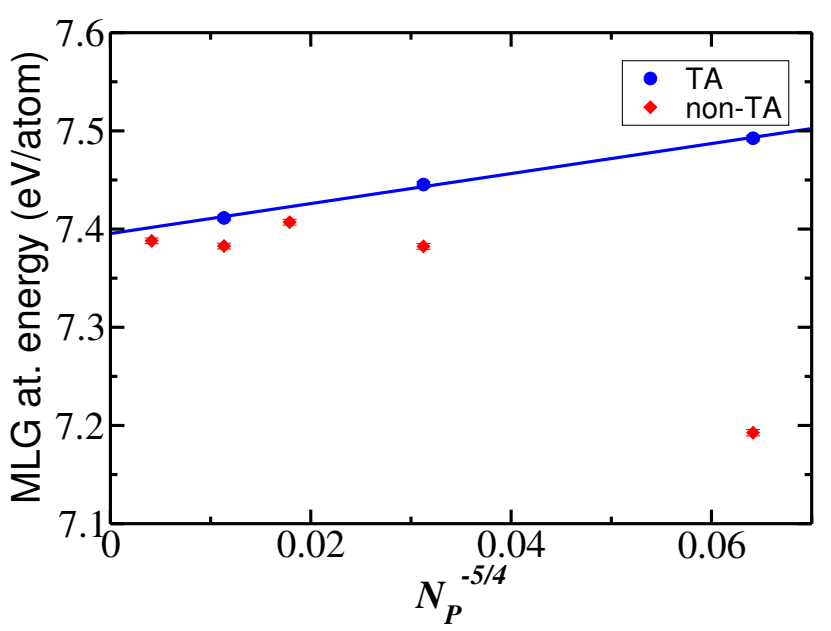

FIG. 1: (Color online) Twist-averaged (TA) and non-TA atomization energies of MLG against $N_{P}^{-5 / 4}$ as calculated by DMC, where $N_{P}$ is the number of primitive cells in the simulation supercell.

DFT-PBE phonon zero-point energy (ZPE) of MLG was calculated using the method of finite displacements in a $6 \times 6$ supercell [43] and found to be $0.165 \mathrm{eV} /$ atom. The $\mathrm{ZPE}$ is a correction to be subtracted from the staticnucleus atomization energy. In principle, an accurate first-principles atomization energy for graphene could be used to estimate the BE of graphite by taking the difference of the experimental atomization energy of graphite [7.371(5) eV/atom 44] and the ZPE-corrected atomization energy of MLG. However, the spread of DFT atomization energies resulting from different choices of pseudopotential (of order 40-70 meV/atom [33]) implies that first-principles pseudopotential calculations cannot currently be used to calculate the BE of graphite by this approach.

Despite a great deal of theoretical and experimental work, the BE of graphene layers remains poorly understood. The cleavage energy of graphite has been measured to be $43(5) \mathrm{meV} /$ atom [14, the BE to be $35(10)$ $\mathrm{meV} /$ atom [45], and the exfoliation energy to be $52(5)$ $\mathrm{meV} /$ atom [46]. More recent experimental work has found the cleavage energy to be $31(2) \mathrm{meV} /$ atom [47. It has been suggested that the latter result may be substantially underestimated, because the experimental data were analyzed using a Lennard-Jones potential, which gives qualitatively incorrect interlayer BEs at large separation [48. Similar difficulties of interpretation may affect the other experimental results in the literature. The results obtained in these works are widely scattered. The DMC method has previously been applied to calculate the BEs of AB- and AA-stacked graphite [49, 50], which were found to be 56(5) and $36(1) \mathrm{meV} /$ atom, respectively, although these calculations were performed in relatively small simulation supercells, and finite-size ef-

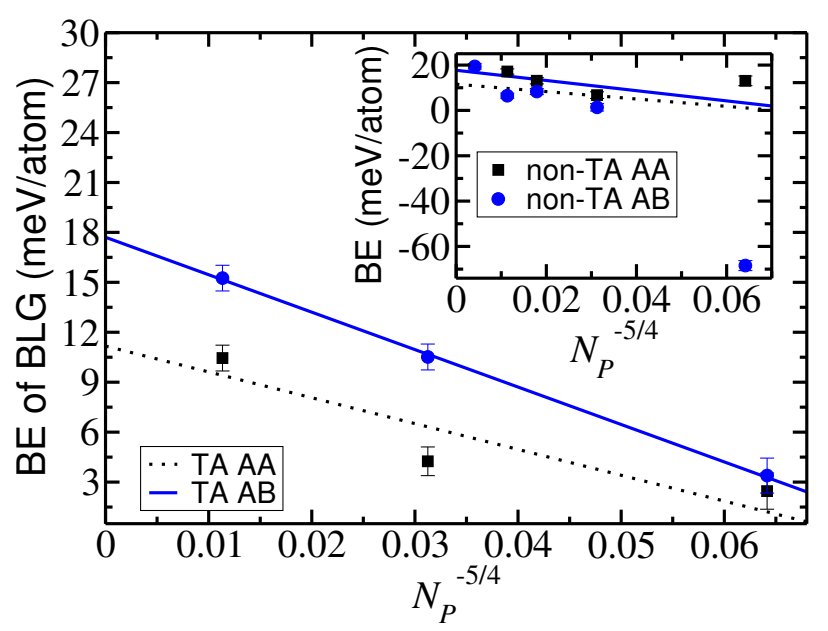

FIG. 2: (Color online) Twist-averaged (TA) BLG BE against $N_{P}^{-5 / 4}$ as calculated by DMC, where $N_{P}$ is the number of primitive cells in the simulation supercell. The inset shows non-twist-averaged BEs. The layer separations are the vdWDF 52 equilibrium values of 3.495 and $3.384 \AA$ for the AAand $\mathrm{AB}$-stacked structures, respectively.

fects may limit the accuracy of the results obtained.

For BLG, we restrict our attention to the nonretarded regime [51, in which the $\mathrm{BE}$ is simply the difference between the nonrelativistic total energy per atom in the monolayer and the bilayer. We used vdW-DF layer separations of $d=3.495 \AA$ and $3.384 \AA$ [52 for the AA- and AB-stacked configurations, respectively. In Fig. 2 we plot the twist-averaged BEs of AA- and AB-stacked BLG as a function of system size. Non-twist-averaged BEs are shown in the inset to Fig. 2 and, as expected, show large oscillations due to momentum-quantization effects. For widely separated graphene layers with nonoverlapping charge densities, single-particle finite-size errors cancel perfectly when the BE is calculated. However, when the layers are closer together, the cancellation is no longer perfect. In practice, near the equilibrium separation, the single-particle errors in the BE correlate closely with the single-particle errors in the total energy of BLG. To evaluate the $\mathrm{BE}$ in the thermodynamic limit, we twistaveraged the BE using Eq. (1) with the BE per atom in place of $E\left(N_{P}, \mathbf{k}_{s}\right)$ and the DFT-LDA total energy per atom of BLG in place of $E_{\mathrm{LDA}}\left(N_{P}, \mathbf{k}_{s}\right)$. We then extrapolated the twist-averaged $\mathrm{BE}$ to infinite system size using Eq. (2). As shown in Fig. 2, the BE of AB-stacked BLG is larger than that of AA-stacked BLG, confirming that the former is the more stable structure.

The area of a simulation cell with $N_{P}$ unit cells is $A=\sqrt{3} N_{P} a^{2} / 2$, where $a$ is the lattice parameter of graphene. If we define the linear size $L$ of the cell via $\pi L^{2}=A$ then we may express the twist-averaged $\mathrm{BE}$ per atom as $\bar{E}_{\text {bind }}(L)=E_{\text {bind }}(\infty)+c^{\prime} L^{-5 / 2}$, where $c^{\prime}$ is $-0.31(5)$ and $-0.43(5) \mathrm{eV} \AA^{5 / 2}$ for the AA-stacked and 
AB-stacked geometries, respectively. The BE is reduced at small supercell sizes $L$. The use of a finite supercell crudely models the situation where the Coulomb interaction between electrons is screened by a metallic substrate. Hence a metallic substrate is expected to weaken the binding of BLG.

In Fig. 3 we plot the BE of AB-stacked BLG against the interlayer separation, as calculated by DFT, DFT-D, and DMC. The layer separations we have studied are not in the asymptotic regime in which the BE falls off as $d^{-3}$, where $d$ is the interlayer separation 53. We have fitted the function $E_{\text {bind }}(d)=A_{4} d^{-4}+A_{8} d^{-8}+A_{12} d^{-12}+$ $A_{16} d^{-16}$ to our DMC BE data, where the $\left\{A_{i}\right\}$ are fitting parameters, which we find to be $A_{4}=-2.9 \times 10^{3} \mathrm{meV}^{4}$, $A_{8}=-2.97 \times 10^{5} \mathrm{meV}^{8}, A_{12}=6.18 \times 10^{7} \mathrm{meV}^{12}$, and $A_{16}=-1.63 \times 10^{9} \mathrm{meV}^{16}$. This function fits the DMC data well, with a $\chi^{2}$ value of 0.007 per data point. The $\mathrm{BE}$ found at the minimum of the fitting curve is $17.8(8)$ $\mathrm{meV} /$ atom at the equilibrium separation of $3.43(4) \AA$. Although the separation that minimizes our fitted $\mathrm{BE}$ curve for AB-stacked BLG is somewhat larger than the separation used in our calculation of the BE reported in Table I, the difference between the BEs is not statistically significant. The Tkatchenko-Scheffler 4. DFT-D scheme shows roughly the same equilibrium separation as DMC, but the magnitude of the $\mathrm{BE}$ is substantially larger. In general, the three DFT-D methods studied [4, 54, 55] disagree with each other and with DMC. Indeed, the magnitude of the $\mathrm{BE}$ (if not the shape of the $\mathrm{BE}$ curve) is best described by the LDA. Our fitted BE curve enables us to calculate the out-of plane zone-center optical phonon frequency $\omega_{\mathrm{ZO}^{\prime}}$ of AB-stacked BLG 56. A comparison of $\omega_{\mathrm{ZO}}$ frequencies obtained by DFT, DMC, and experiment [57 is shown in Table II. Our DFT-LDA frequency is in reasonable agreement with the result $(76.8$ $\mathrm{cm}^{-1}$ ) reported in Ref. [58. The difference between the $\omega_{\mathrm{ZO}}$ frequency predicted by our fit to our DMC data and the experimental result is negligible $\left[3(7) \mathrm{cm}^{-1}\right] 33$.

In summary, we have used the DMC method to determine the BE of BLG. Our approach includes a full, first-principles treatment of vdW interactions. We have found the static-nucleus atomization energy of MLG to be $7.395(3) \mathrm{eV} /$ atom, although the uncertainty in this result due to the use of nonlocal pseudopotentials may be as much as $70 \mathrm{meV} /$ atom 33 . We find the BEs of AA- and AB-stacked BLG near their equilibrium separations to be $11.5(9)$ and $17.7(9) \mathrm{meV} /$ atom, respectively. Our results indicate that current DFT-D and vdW-DF methods significantly overbind 2D materials.

We acknowledge financial support from the UK Engineering and Physical Sciences Research Council (EPSRC). This work made use of the facilities of Lancaster University's High-End Computing facility, N8 HPC provided and funded by the N8 consortium and EPSRC (Grant No. EP/K000225/1), and the ARCHER UK National Supercomputing Service. We would like to thank

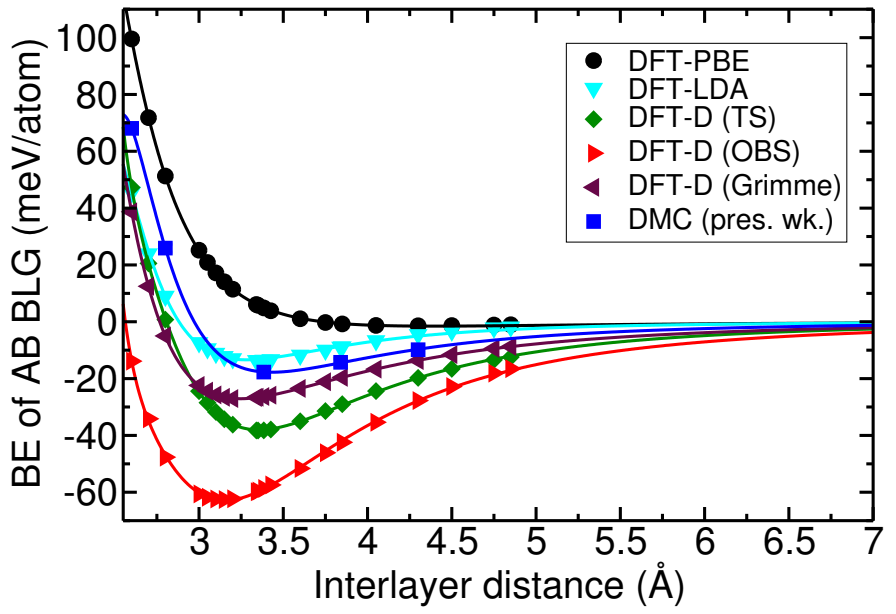

FIG. 3: (Color online) BE curve of AB-stacked BLG as a function of interlayer distance calculated using DFT, DFT$\mathrm{D}$, and DMC methods. Our DFT-D calculations used the Tkatchenko-Scheffler (TS) 4], Ortmann-Bechstedt-Schmidt (OBS) 54, and Grimme 55 vdW corrections.

TABLE II: The equilibrium separation $d_{0}$, static-lattice BE at equilibrium separation, and out-of-plane zone-center opticalphonon frequency $\omega_{\mathrm{ZO}}$ of AB-stacked BLG obtained by DFT, DFT-D, DMC, and experiment. The minimum of the curve fitted to the DMC BE data, which is reported in this table, is in statistical agreement with the DMC BE obtained using a fixed layer separation of $3.384 \AA$, which is reported in Table I

\begin{tabular}{llll}
\hline \hline Method & $d_{0}(\AA)$ & $\mathrm{BE}(\mathrm{meV} /$ at. $)$ & $\omega_{\mathrm{ZO}^{\prime}}\left(\mathrm{cm}^{-1}\right)$ \\
\hline DFT-PBE & 4.40 & 1.53 & 16 \\
DFT-LDA & 3.28 & 13.38 & 84 \\
DFT-D (TS) & 3.35 & 38.03 & 111 \\
DFT-D (OBS) & 3.15 & 62.70 & 133 \\
DFT-D (Grimme) & 3.25 & 27.08 & 95 \\
DMC (pres. wk.) & $3.43(4)$ & $17.8(8)$ & $83(7)$ \\
Exp. [57] & & & $80(2)$ \\
Exp. [59] & & & $89.7(15)$ \\
\hline \hline
\end{tabular}

S. Milana and A. C. Ferrari for providing their experimental phonon data.

[1] S. Grimme, J. Comput. Chem. 25, 1463 (2004); S. Grimme, J. Comput. Chem. 27, 1787 (2006).

[2] M. Hasegawa, K. Nishidate, and H. Iyetomi, Phys. Rev. B 76, 115424 (2007).

[3] S. Grimme et al., J. Chem. Phys. 132, 154104 (2010).

[4] A. Tkatchenko and M. Scheffler, Phys. Rev. Lett. 102, 073005 (2009).

[5] H. Rydberg et al., Phys. Rev. Lett. 91, 126402 (2003).

[6] M. Dion, H. Rydberg, E. Schroder, D.C. Langreth, and B. I. Lundqvist, Phys. Rev. Lett. 92, 246401 (2004); M. 
Dion, H. Rydberg, E. Schroder, D.C. Langreth, and B.I. Lundqvist, Phys. Rev. Lett. 95, 109902(E) (2005).

[7] S.D. Chakarova-Kack, E. Schroder, B.I. Lundqvist, and D.C. Langreth, Phys. Rev. Lett. 96, 146107 (2006).

[8] T. Thonhauser et al., Phys. Rev. B 76, 125112 (2007).

[9] S. Lebègue et al., Phys. Rev. Lett. 105, 196401 (2010).

[10] T. Olsen and K.S. Thygesen, Phys. Rev. B 87, 075111 (2013).

[11] H. Eshuis, J.E. Bates, and F. Furche, Theor. Chem. Acc. 131, 1084 (2012).

[12] X. Ren et al., J. Mater Sci. 47, 7447 (2012).

[13] B. Jeziorski, R. Moszynski, and K. Szalewicz, Chem. Rev. 94, 1887 (1994).

[14] L.A. Girifalco and R.A. Lad, J. Chem. Phys. 25, 693 (1956).

[15] L.A. Girifalco, M. Hodak, R.S. Lee, Phys. Rev. B 62, 13104 (2000).

[16] J.F. Dobson, A. White, and A. Rubio, Phys. Rev. Lett. 96, 073201 (2006).

[17] I.V. Lebedeva et al., Phys. Chem. Chem. Phys. 13, 5687 (2011).

[18] Y.J. Dappe et al., J. Phys.: Condens. Matter 24, 424208 (2012).

[19] R. Podeszwa, J. Chem. Phys. 132, 044704 (2010).

[20] T. Gould, S. Lebègue, and J.F. Dobson, J. Phys.: Condens. Matter 25, 445010 (2013).

[21] W. Gao and A. Tkatchenko, Phys. Rev. Lett. 114, 096101 (2015).

[22] R.J. Needs et al., J. Phys.: Condens. Matter 22, 023201 (2010).

[23] D.M. Ceperley and B.J. Alder, Phys. Rev. Lett. 45, 566 (1980).

[24] W.M.C. Foulkes et al., Rev. Mod. Phys. 73, 33 (2001).

[25] J.B. Anderson, J. Chem. Phys. 65, 4121 (1976).

[26] C.-R. Hsing et al., New J. Phys. 16, 113015 (2014).

[27] S.J. Clark et al., Z. Kristallogr. 220, 567 (2005).

[28] J.P. Perdew, K. Burke, and M. Ernzerhof, Phys. Rev. Lett. 77, 3865 (1996).

[29] D. Alfè and M.J. Gillan, Phys. Rev. B 70, 161101 (2004).

[30] N.D. Drummond, M.D. Towler, and R.J. Needs, Phys. Rev. B 70, 235119 (2004).

[31] N.D. Drummond and R.J. Needs Phys. Rev. B 72, 085124 (2005).

[32] C.J. Umrigar, K.G. Wilson, and J.W. Wilkins, Phys. Rev. Lett. 60, 1719 (1988).

[33] See Supplemental Material [url], which includes Refs. 60 66.

[34] J.R. Trail and R.J. Needs, J. Chem. Phys. 122, 014112 (2005).

[35] J.R. Trail and R.J. Needs, J. Chem. Phys. 122, 174109 (2005).

[36] D.E. Parry, Surf. Sci. 49, 433 (1975); erratum, Surf. Sci. 54, 195 (1976)

[37] B. Wood et al., J. Phys.: Condens. Matter 16, 891 (2004).

[38] N.D. Drummond, R.J. Needs, A. Sorouri, and W.M.C. Foulkes, Phys. Rev. B 78, 125106 (2008).

[39] G. Gómez-Santos, Phys. Rev. B, 80, 245424 (2009).

[40] G.L. Klimchitskaya and V.M. Mostepanenko, Phys. Rev.
B, 87, 075439 (2013).

[41] C. Lin, F.H. Zong, and D.M. Ceperley, Phys. Rev. E 64, 016702 (2001).

[42] H. Shin et al., J. Chem. Phys. 140, 114702 (2014).

[43] K. Refson, P.R. Tulip, and S.J. Clark, Phys. Rev. B 73, 155114 (2006).

[44] CRC Handbook of Chemistry and Physics 91st Edition, W.M. Haynes, (Ed) CRC Press, Inc., Roca Raton, 20102011, p. 5-1.

[45] L.X. Benedict et al., Chem. Phys. Lett. 286, 490 (1998).

[46] R. Zacharia, H. Ulbricht, and T. Hertel, Phys. Rev. B 69, 155406 (2004)

[47] Z. Liu et al., Phys. Rev. B 85, 205418 (2012).

[48] T. Gould et al., J. Chem. Phys. 139, 224704 (2014).

[49] L. Spanu, S. Sorella, and G. Galli, Phys. Rev. Lett. 103, 196401 (2009).

[50] P. Ganesh et al., J. Chem. Theory Comput. 10, 5318 (2014).

[51] At large separations the use of the static Coulomb interaction between electrons in two graphene layers ceases to be valid due to the finite speed of light, resulting in a crossover to a regime in which the attractive forces arise from photon zero-point energy 67.

[52] I. Brihuega et al., Phys. Rev. Lett. 109, 196802 (2012).

[53] J.F. Dobson, T. Gould, and G. Vignale, Phys. Rev. X 4, $021040(2014)$.

[54] F. Ortmann, F. Bechstedt, and W.G. Schmidt, Phys. Rev. B 73, 205101 (2006).

[55] S. Grimme, J. Comput. Chem. 27, 1787, (2006).

[56] The interlayer BE per atom of BLG can be written as $E_{\text {bind }}(d)=E_{0}+\frac{1}{8} m_{C} \omega_{\mathrm{ZO}^{\prime}}^{2}\left(d-d_{0}\right)^{2}+O\left(d-d_{0}\right)^{3}$, where $E_{0}$ is the $\mathrm{BE}$ per atom at the equilibrium separation $d_{0}$, $m_{C}$ is the mass of a carbon atom, and $\omega_{\mathrm{ZO}^{\prime}}$ is the outof-plane phonon frequency.

[57] C.H. Lui and T.F. Heinz, Phys. Rev. B 87, 121404(R) (2013).

[58] S.K. Saha, U.V. Waghmare, H.R. Krishnamurthy, and A.K. Sood, Phys. Rev. B 78, 165421 (2008).

[59] A. Ferrari and S. Milana (unpublished) - presented at Graphene Week Conference 2015.

[60] L. Mitáš, E.L. Shirley, and D.M. Ceperley, J. Chem. Phys. 95, 3467 (1991).

[61] M. Hasegawa and K. Nishidate, Phys. Rev. B 70, 205431 (2004).

[62] A. Hansson, F. de Brito Mota, and R. Rivelino, Phys. Rev. B 86, 195416 (2012).

[63] G. Graziano et al., J. Phys.: Condens. Matter 24, 424216 (2012).

[64] N. Troullier and J.L. Martins, Phys. Rev. B 43, 1993 (1991).

[65] M. Burkatzki, C. Filippi, and M. Dolg, J. Chem. Phys. 126, 234105 (2007).

[66] C.J. Umrigar, M.P. Nightingale, and K.J. Runge, J. Chem. Phys. 99, 2865 (1993).

[67] H.B.G. Casimir and D. Polder, Phys. Rev. 73, 360 (1948). 


\title{
Quantum Monte Carlo Calculation of the Binding Energy of Bilayer Graphene: Supplemental Material
}

\author{
E. Mostaani and N. D. Drummond \\ Department of Physics, Lancaster University, Lancaster LA1 4YB, United Kingdom \\ V. I. Fal'ko \\ Department of Physics, Lancaster University, Lancaster LA1 4 YB, United Kingdom and \\ National Graphene Institute, University of Manchester, \\ Booth Street East, Manchester M13 9PL, United Kingdom
}

\section{ATOMIZATION ENERGY OF MONOLAYER GRAPHENE}

Table I compares the atomization energies of monolayer graphene predicted by density functional theory (DFT) with different functionals and by diffusion quantum Monte Carlo (DMC). Our DFT static-nucleus atomization energies were obtained using the local density approximation (LDA) and Perdew-Burke-Ernzerhof (PBE) functionals with both ultrasoft [1] and Dirac-Fock pseudopotentials 2] using a plane-wave cutoff energy of 220 Ry. Our DMC calculations used the pseudopotential locality approximation [3]. We compare our results with previous results in the literature [4, 5. Both DFT-PBE and DFT-LDA calculations overestimate the atomization energy, but the error in the LDA result is significantly larger.

TABLE I: Static-nucleus atomization energy $E_{\text {atom }}$ of monolayer graphene obtained in various DFT and DMC studies. The bond lengths quoted in the table are the ones used in the calculations, not necessarily the optimized bond length for the given method.

\begin{tabular}{llcc}
\hline \hline Method & Pseudopotential & Bond length $(\AA)$ & $E_{\text {atom }}(\mathrm{eV} /$ atom $)$ \\
\hline DFT-LDA [6] & & 1.412 & 8.96 \\
DFT-LDA [4] & & 1.420 & 8.873 \\
DFT-LDA (pres. wk.) & Ultrasoft [1] & 1.420 & 8.632 \\
DFT-LDA (pres. wk.) & Dirac-Fock [2] & 1.420 & 8.578 \\
DFT-PBE [5] & Norm-conserving [7] & 1.440 & 7.847 \\
DFT-PBE [6] & & 1.424 & 7.93 \\
DFT-PBE [] & Dirac-Fock [9] & 1.421 & 7.906 \\
DFT-PBE (pres. wk.) & Ultrasoft [1] & 1.420 & 7.873 \\
DFT-PBE (pres. wk.) & Dirac-Fock [2] & 1.420 & 7.837 \\
DMC [] & Dirac-Fock [9] & 1.421 & $7.464(10)$ \\
DMC (pres. wk.) & Dirac-Fock [2] & 1.420 & $7.395(3)$ \\
\hline \hline
\end{tabular}

The DFT results in Table [ show that using different pseudopotentials changes the calculated atomization energy of graphene by 40-70 meV/atom, which is very large on the scale of the binding energy (BE) of graphite. However, DFTLDA and DFT-D calculations at different layer separations show that both ultrasoft and Dirac-Fock pseudopotentials give the same value for the BE of AB-stacked bilayer graphene (BLG): see Table II. The cancellation of pseudopotential errors between bilayer and monolayer graphene is much larger than between monolayer graphene and an isolated $\mathrm{C}$ atom, so our calculation of the BE of BLG is expected to be significantly more accurate than our calculation of the atomization energy of graphene. It should be noted that pseudopotential errors are at least as bad in DMC calculations as in DFT; although DMC is a highly accurate method, it cannot do better than permitted by the pseudopotentials used to model atoms.

\section{FINITE-POPULATION ERRORS IN OUR DIFFUSION MONTE CARLO DATA}

We have carried out calculations to investigate finite-population errors 13 in our DMC calculations. Figures 1(a) and 1(b) show the non-twist-averaged DMC ground-state energy per atom for a $3 \times 3$ supercell of monolayer graphene and the DMC energy of an isolated, spin-polarized $\mathrm{C}$ atom against the reciprocal of the target configuration population. The DMC energies have been extrapolated linearly to zero time step in each case. The function fitted 
TABLE II: Comparison of DFT and DFT-D BEs of AB-stacked BLG at equilibrium separation $3.384 \AA$ using ultrasoft and Dirac-Fock pseudopotentials.

\begin{tabular}{llc}
\hline \hline Method & Pseudopotential & BE (meV/atom) \\
\hline DFT-PBE & Dirac-Fock [2] & 6.03 \\
DFT-PBE & Ultrasoft 1] & 4.87 \\
DFT-LDA & Dirac-Fock [2] & 12.39 \\
DFT-LDA & Ultrasoft [1] & 13.53 \\
DFT-D (TS) [10] & Dirac-Fock [2] & 38.35 \\
DFT-D (TS) [10] & Ultrasoft [1] & 38.22 \\
DFT-D (OBS) [1] & Dirac-Fock [2] & 59.32 \\
DFT-D (OBS) [1] & Ultrasoft 1] & 59.58 \\
DFT-D (Grimme) [12 & Dirac-Fock [2] & 27.01 \\
DFT-D (Grimme) [12] & Ultrasoft 1] & 26.57 \\
\hline \hline
\end{tabular}

to the DMC data in Fig. 1 is $E\left(N_{\text {pop }}\right)=E(\infty)+B / N_{\text {pop }}$, where $N_{\text {pop }}$ is the target configuration population [13. For our Slater-Jastrow trial wave function, we find that $B=1.4(6) \mathrm{eV}$ for monolayer graphene in a $3 \times 3$ supercell. The gradient $B$ is of marginal significance. For populations in excess of 512 configurations the expected bias in the DMC energy is less than 2.7(12) meV/atom. We used target populations of 1024 configurations in our production calculations for supercells of $3 \times 3$ primitive cells and target populations of 512 configurations for larger supercells. Population-control biases are always positive and must largely cancel out of the BE of BLG. For an isolated C atom, the value of $B$ is not statistically significant. We have used a target population of 1024 configurations in our calculation for the $\mathrm{C}$ atom; the resulting population-control bias in the DMC energy is less than $1 \mathrm{meV}$.
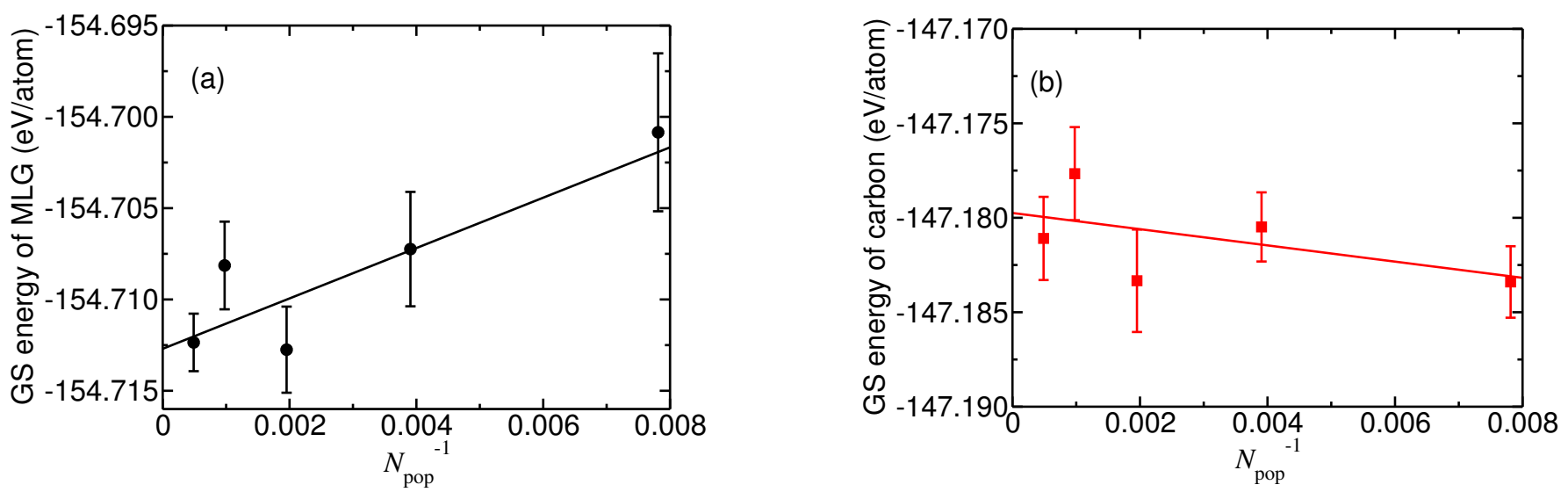

FIG. 1: (a) Non-twist-averaged DMC ground-state (GS) energy of a $3 \times 3$ cell of monolayer graphene and (b) DMC GS energy of a $\mathrm{C}$ atom as a function of the reciprocal of the configuration population $N_{\text {pop }}^{-1}$.

\section{TIME-STEP ERRORS IN OUR DIFFUSION MONTE CARLO DATA}

Figure 2(a) shows the non-twist-averaged ground-state DMC energies of $3 \times 3$ supercells of monolayer graphene and AB-stacked BLG as a function of time step. Linear extrapolations to zero time step using time steps of 0.01 and 0.04 a.u. are shown by dashed lines. The differences between the results of linear extrapolation using the time steps 0.01 and $0.04 \mathrm{a}$ a. and those obtained using three time steps $0.001,0.005$, and 0.01 a.u. are $11(3)$ and $13(3) \mathrm{meV} / \mathrm{atom}$ for the bilayer and monolayer, respectively. These differences indicate the magnitude of the error in the atomization energy due to residual time-step bias.

Figure 2(b) shows the non-twist-averaged DMC BE of a $3 \times 3$ cell of AB-stacked BLG against time step. Unlike the total energy, the BE varies linearly with time step across the range time steps considered. The difference between the extrapolated BE using time steps of 0.01 and 0.04 a.u. and that extrapolated using all the time steps shown in 
Fig. 2(b) is 1(2) meV/atom, which is negligible. Time-step bias largely cancels between the bilayer and monolayer energies, and the remaining bias in the BE may easily be removed by linear extrapolation to zero time step.
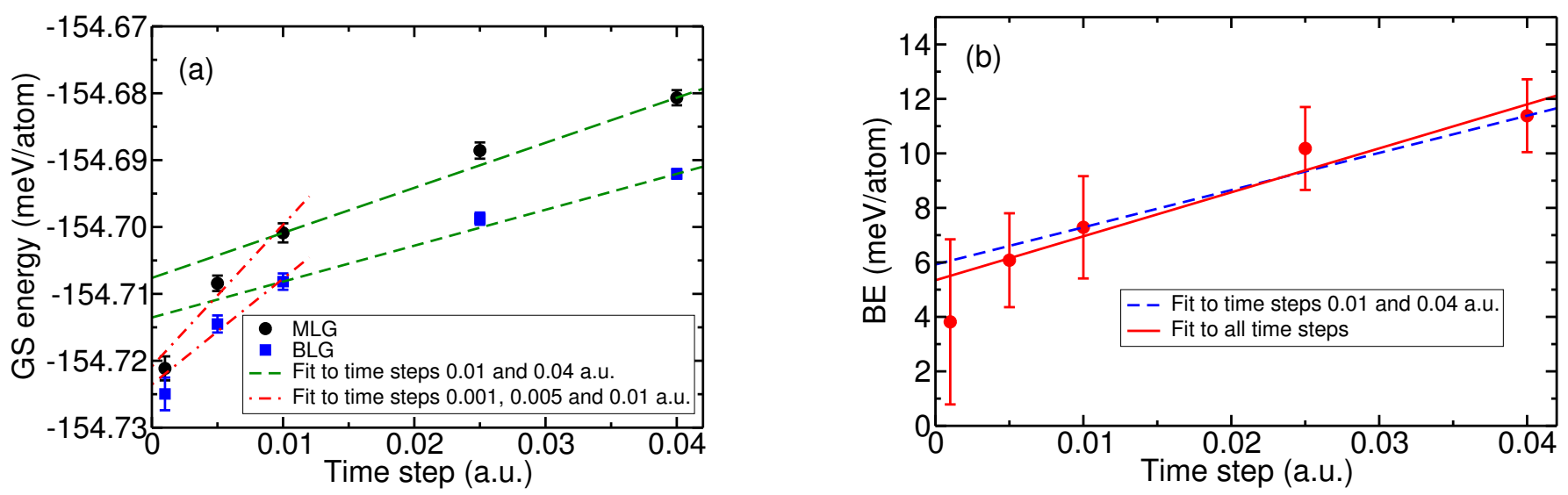

FIG. 2: (a) Non-twist-averaged DMC energy of a $3 \times 3$ cell of monolayer graphene (MLG) and AB-stacked BLG as a function of time step. (b) Non-twist-averaged DMC BE of a $3 \times 3$ cell of AB-stacked BLG against time step.

\section{CHOICE OF FITTING FUNCTION FOR THE BINDING-ENERGY CURVE}

We have investigated different fitting functions for our DMC BE data for AB-stacked BLG. In Fig. 3 we compare the following fits to the DMC BE:

Fit 1a The maroon long-dashed line shows a fit of

$$
E_{\text {bind }}(d)=a+b\left(d-d_{0}\right)^{2}+c\left(d-d_{0}\right)^{3}
$$

to the DMC BE data at interlayer separations $d=2.8,3.384$, and $3.84 \AA$, where $a, b$, and $c$ are fitting parameters and $d_{0}=3.384 \AA$ is fixed at the vdW-DF interlayer equilibrium separation [14].

Fit 1 b The red short-dashed line shows a fit of Eq. (1) to the DMC BE data at interlayer separations $d=2.6,2.8$, 3.384 , and $3.84 \AA$. This time $a, b, c$, and $d_{0}$ are all fitting parameters.

Fit 1c The green dot-dashed line shows a fit of Eq. (1) to the DMC BE data at interlayer separations $d=2.8,3.384$, 3.84, and $4.3 \AA$. Again, $a, b, c$, and $d_{0}$ are all fitting parameters.

Fit 1d The blue dash-double dotted line shows a fit of Eq. (1) to all our DMC BE data. Again, $a, b, c$, and $d_{0}$ are all fitting parameters.

Fit 2 The solid black line shows a fit of

$$
E_{\text {bind }}(d)=\alpha \exp (-\beta d)+\gamma d^{-4}
$$

to all our DMC BE data, where $\alpha, \beta$, and $\gamma$ are fitting parameters [15].

Fit 3 The solid magenta line shows a fit of

$$
E_{\text {bind }}(d)=A_{4} d^{-4}+A_{8} d^{-8}+A_{12} d^{-12}+A_{16} d^{-16}
$$

to all our DMC BE data, where the $\left\{A_{i}\right\}$ are fitting parameters.

Equation (3) has the correct $d^{-4}$ form of decay for the BE at intermediate range and has a reasonable model of the hard-core repulsion. The $\chi^{2}$ values obtained with Fits 2 and 3 are 1.3 and 0.007 per data point, respectively, compared with 0.4 per data point for Fit 1 , which has the same number of fitting parameters as Fit 3 . The $\chi^{2}$ value per data point for Fits $1 \mathrm{a}-1 \mathrm{c}$ is zero, because the number of data points is equal to the number of parameters. Fit 2 shows unphysical behavior: the exponential term prefers to be attractive while the $d^{-4}$ tries to be repulsive. We have therefore used Fit 3 to obtain the breathing-mode frequency presented in the main body of our article. 


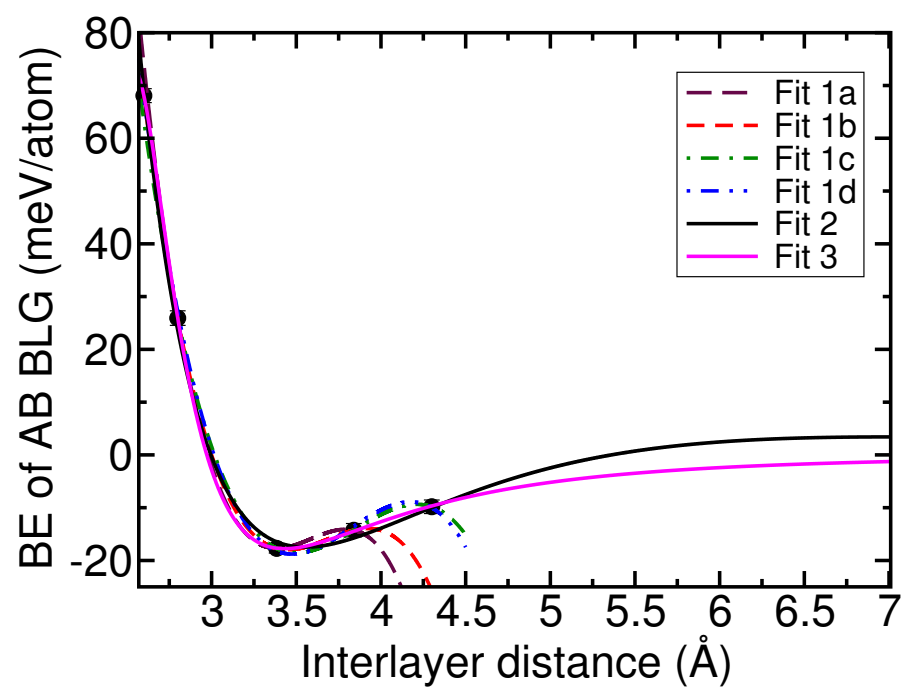

FIG. 3: DMC BE of AB-stacked BLG against interlayer separation using different fitting curves.

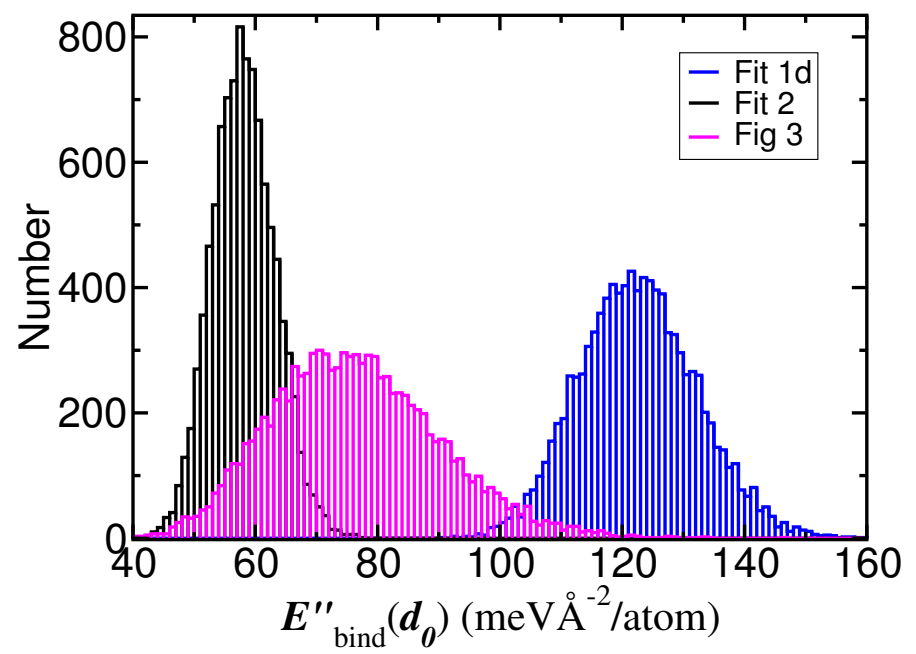

FIG. 4: Histogram of $E_{\text {bind }}^{\prime \prime}\left(d_{0}\right)$ obtained in bootstrap Monte Carlo sampling of Fits 1d, 2, and 3 with 10,000 samples.

In Table III we compare the equilibrium separation $d_{0}$, the corresponding BE $E_{\text {bind }}\left(d_{0}\right)$, the curvature $E_{\text {bind }}^{\prime \prime}\left(d_{0}\right)$, and the out-of-plane optical phonon frequency (the breathing mode $\mathrm{ZO}^{\prime}$ ) obtained with the different fits to our DMC BE data.

To evaluate error bars on quantities such as the second derivative of the BE at the minimum and the corresponding phonon frequency, we used bootstrap Monte Carlo sampling of our data together with repeated $\chi^{2}$ fits. In Fig. 4 , histograms of $E_{\mathrm{bind}}^{\prime \prime}\left(d_{0}\right)$ for Fits $1 \mathrm{~d}, 2$ and 3 are shown. The phonon frequencies obtained using Fits 2 and 3 are in good agreement, although the difference between Fits $1 \mathrm{~d}$ and 3 is more significant. However, we believe Fit 3 to be more reliable because it is constructed to have the correct asymptotic behavior.

[1] S.J. Clark et al., Z. Kristallogr. 220, 567 (2005).

[2] J.R. Trail and R.J. Needs, J. Chem. Phys. 122, 014112 (2005).

[3] L. Mitáš, E.L. Shirley, and D.M. Ceperley, J. Chem. Phys. 95, 3467 (1991).

[4] M. Hasegawa and K. Nishidate, Phys. Rev. B 70, 205431 (2004).

[5] A. Hansson, F. de Brito Mota, and R. Rivelino, Phys. Rev. B 86, 195416 (2012).

[6] G. Graziano et al., J. Phys.: Condens. Matter 24, 424216 (2012). 
TABLE III: Equilibrium separation $d_{0}$ and corresponding BE $E_{\text {bind }}\left(d_{0}\right)$, second derivative of the BE at the minimum $E_{\text {bind }}^{\prime \prime}\left(d_{0}\right)$, and out-of-plane phonon frequency $\omega_{\mathrm{ZO}^{\prime}}$ that are obtained by fitting different curves to our DMC data for the BE of BLG. Experimental results [16] are shown for comparison.

\begin{tabular}{llccc}
\hline \hline Fit & $d_{0}(\AA)$ & $E_{\text {bind }}\left(d_{0}\right)(\mathrm{meV} /$ atom $)$ & $E_{\text {bind }}^{\prime \prime}\left(d_{0}\right)\left(\mathrm{meV} \AA^{-2} /\right.$ atom $)$ & $\omega_{\mathrm{ZO}^{\prime}}\left(\mathrm{cm}^{-1}\right)$ \\
\hline Fit 1a & 3.384 & $-17.7(9)$ & $131(11)$ & $109(4)$ \\
Fit 1b & $3.45(4)$ & $-18(1)$ & $110(17)$ & $100(8)$ \\
Fit 1c & $3.50(4)$ & $-18.6(8)$ & $109(12)$ & $99(7)$ \\
Fit 1d & $3.48(3)$ & $-18.8(8)$ & $122(9)$ & $105(4)$ \\
Fit 2 & $3.55(2)$ & $-17.5(7)$ & $58(5)$ & $72(3)$ \\
Fit 3 & $3.43(4)$ & $-17.8(8)$ & $76(13)$ & $83(7)$ \\
Experiment [16] & & $70(4)$ & $80(2)$ \\
Experiment [17] & & $89(3)$ & $89.7(15)$ \\
\hline \hline
\end{tabular}

[7] N. Troullier and J.L. Martins, Phys. Rev. B 43, 1993 (1991).

[8] H. Shin et al., J. Chem. Phys. 140, 114702 (2014).

[9] M. Burkatzki, C. Filippi, and M. Dolg, J. Chem. Phys. 126, 234105 (2007).

[10] A. Tkatchenko and M. Scheffler, Phys. Rev. Lett. 102, 073005 (2009).

[11] F. Ortmann, F. Bechstedt, and W.G. Schmidt, Phys. Rev. B 73, 205101 (2006).

[12] S. Grimme, J. Comput. Chem. 27, 1787, (2006).

[13] C.J. Umrigar, M.P. Nightingale, and K.J. Runge, J. Chem. Phys. 99, 2865 (1993).

[14] I. Brihuega et al., Phys. Rev. Lett. 109, 196802 (2012).

[15] L. Spanu et al., Phys. Rev. Lett. 103, 196401 (2009).

[16] C.H. Lui and T.F. Heinz, Phys. Rev. B 87, 121404(R) (2013).

[17] A. Ferrari and S. Milana (unpublished) - presented at Graphene Week Conference 2015. 